\title{
AN APPLICATION OF THE GARCH-t MODEL ON CENTRAL EUROPEAN STOCK RETURNS
}

\author{
Miloslav VOŠVRDA, Filip ŽlKEŠ *
}

\begin{abstract}
:
The purpose of this paper is to investigate the time-series and distributional properties of Central European stock returns. We test the random walk hypothesis and then consider an alternative to random walk - the ARIMA model for stock prices. The behavior of volatility of returns over time is studied using the GARCH-t model which also allows us to learn more about the distribution properties of stock returns. We employ the BDS test to assess the ability of the estimated GARCH- $t$ model to capture all nonlinearities in stock returns. Our empirical findings reveal that the Czech and Hungarian stock market indices are predictable from the time series of historical prices, whereas that of Poland is not. The returns on all three indices are conditionally heteroskedastic and non-normal. The estimated number of degrees of freedom ranges from 18 to 4 .
\end{abstract}

Keywords: conditional heteroskedasticity, GARCH, leptokurtosis, market efficiency

JEL Classification: C32, G15

\section{Introduction}

One of the main principles of modern finance is that expected return is somehow related to risk. There are numerous equilibrium models that specify this relationship (see, for example, Cochrane, 2001 for details). For instance, in the standard Sharpe-Lintner-Mossin CAPM, expected return is proportional to systematic risk, i.e. to risk that cannot be diversified away. Although there is not a general agreement in the literature as to what model explains the reality best, it is widely accepted that when agents are risk-averse expected return is an increasing function of risk.

A widely used measure of the risk of an asset is the standard deviation of returns from the unconditional mean. This measure can be loosely interpreted as longrun volatility, since it appears to be determined by various economic fundamental characteristics for that particular asset and is usually assumed constant for the pe-

*) Academy of Sciences of the Czech Republic, Dept. of Econometrics and Charles University, Faculty of Social Sciences, Institute of Economic Studies, Opletalova 26, CZ - 11000 Prague 1 (e-mail: vosvrda@utia.cas.cz; zikesf@yahoo.com).

**) This research was supported by the Grant Agency of Charles University under grant GA UK 287/ 2003/A-EK/FSV, and by the Grant Agency of the Czech Republic under grants 402/01/0034 and 402/04/ 1294. 
riod under study. It has been observed in numerous studies, however, that returns are not homoskedastic and that "large price changes tend to be followed by large changes of either sign and small changes tend to be followed by small changes of either sign"(Mandelbrot, 1963, p. 418). Also it has been observed empirically that the unconditional distributions of stock returns exhibit fatter tails (see, for example, Fama, 1965) than what would be consistent with the normal distribution. But the distributional properties of stock returns have important implications for asset pricing: in the above mentioned CAPM model, for example, the variances and covariances are used as a measure of the risk, but as Bollerslev (1987, p. 542) notes, "depending on the distribution of the returns, the variance may not be a valid or sufficient statistic to use". Another example of the importance of the distributional assumptions is the well-known Black-Scholes option pricing model which rests on the hypothesis of normality of the underlying stock returns and assumes constant volatility of returns over the life of the option. Clearly, correctly specifying the distribution of asset returns is a necessary condition for rational asset pricing.

Although the literature on conditional heteroskedasticity of stock returns is fairly voluminous, only a few applications to emerging stock markets in Central Europe have been published to date. Vošvrda et al. (1998) investigate the time series properties of the Czech PX-50 index and find significant conditional heteroskedasticity in its daily returns. Similarly, Gilmore and McManus (2001) find evidence of timevarying volatility in weekly returns on major Central European stock indices. Both studies, however, assume conditional normality of stock returns rather than investigating the distributions empirically. In this paper, we use Student's $t$-distribution with $v$ degrees of freedom, where $v$ is a parameter to be estimated from the data. As we will see below, this approach yields a better fit and hence the resulting GARCH parameter estimates are more efficient.

This paper is organized as follows. Next section presents a brief description of the methodology applied. Section 3 contains the data description and in Section 4 the empirical results are reported. The paper concludes with a short summary and suggestions for future research in Section 5.

\section{Methodology}

In this paper we focus on the Generalized Autoregressive Conditional Heteroskedastic Model (GARCH) developed in Bollerslev (1986). The vast majority of empirical studies use the method of maximum likelihood to estimate the parameters of a $\mathrm{GARCH}$ model. The usual assumption is made that the error term is conditionally normally distributed, e.g. $\varepsilon_{t} \sim N\left(0, h_{t}\right)$. Then the maximum likelihood estimator is consistent and asymptotically normally distributed even if the true distribution is nonnormal. The standard errors of the estimates, however, are inconsistent if the true distribution is not normal, but Bollerslev and Wooldridge (1988) derive a robust estimator of the covariance matrix that gives consistent estimates of the standard errors under such circumstances. This method is called the quasi-maximum likelihood estimation (QMLE). Alternatively, one can assume a different conditional distribution for the error term. Following Bollerslev (1987) we assume that $u_{t}$ is conditionally $t$-distributed with $v$ degrees of freedom, i.e.

$$
\left(\varepsilon_{t} \mid h_{t}\right) \sim f_{v}\left(\varepsilon_{t} \mid h_{t}\right)=\Gamma\left(\frac{v+1}{2}\right) \Gamma\left(\frac{v}{2}\right)^{-1}\left((v-2) h_{t}\right)^{-1 / 2}\left(1+\frac{\varepsilon_{t}^{2}}{h_{t}(v-2)}\right)^{-(v+1) / 2}, v>2
$$

It is well-known property of the Student's $t$-distribution that as the number of the degrees of freedom increases without bound, the $t$-distribution approaches a nor- 
mal distribution with zero mean and variance $h_{t}$, but for finite $v$, the $t$-distribution has fatter tails than the corresponding normal distribution. Hence assuming the conditional $t$-distribution in connection with a GARCH model may better account for the excess kurtosis found in the stock market returns. Moreover, the estimated number of degrees of freedom, $\hat{v}$ may indicate the source of the excess kurtosis in the unconditional stock returns: according to Connolly (1992), if $\hat{v}<10$, both non-normality and conditional heteroskedasticity explain the excess kurtosis in returns, whereas if $\hat{v}>30$ conditional heteroskedasticity is the only source of fat tails in the unconditional distribution of returns.

Since the application of a GARCH model requires a properly specified equation for the mean of returns, we first test the random walk hypothesis using a heteroskedasticity-consistent variance ratio test due to Lo and MacKinlay (1988). When the null hypothesis of random walk can be rejected we consider an ARMA model as an alternative for modelling the stock returns. The usual Box-Jenkins methodology is applied to identify the model. The behavior of volatility of returns over time is then studied within the context of the appropriate mean equation using the simple $\mathrm{GARCH}(1,1)-t$ model, which is estimated by maximum likelihood. Our model is thus summarized by the following equations:

$$
\begin{aligned}
& r_{t}=\mu+\phi_{1} r_{t-1}+\ldots+\phi_{p} r_{t-p}+\varepsilon_{t}+\theta_{1} \varepsilon_{t-1}+\ldots+\theta_{q} \varepsilon_{t-q}, \\
& \varepsilon_{t}=u_{t} \sqrt{h_{t}}, \\
& h_{t}=\omega+\delta_{1} h_{t-1}+\alpha_{1} \varepsilon_{t-1}^{2}
\end{aligned}
$$

where $r_{t}$ denotes the one-period rate of return on a market index and $\left\{u_{t}\right\}$ is a white noise process with unit variance. We also test for two alternatives to the simple GARCH model: the threshold ARCH (TARCH) developed by Zakoian (1994) and Glosten et al. (1993) and the ARCH-in-mean suggested by Engle et al. (1987). Finally, we employ the BDS test (see Brock et al., 1987) to asses the ability of the estimated GARCH- $t$ model to capture all nonlinearities in stock returns.

The literature on GARCH modelling of stock returns from developed markets is voluminous (see Bollerslev et al., 1992, for an overview). The same, however, does not hold for emerging market in Central Europe. In the majority of empirical studies concerning emerging Central European stock markets, the focus is on market efficiency and stock return predictability using test procedures corrected for general forms of heteroskedasticity (e.g. Filer and Hanousek, 1996; Gilmore and McManus, 2001). Only in Vošvrda et al. (1998), the behavior of volatility is studied in greater detail, but the analysis is confined to the Czech stock market index, PX-50. Hence, in this paper, we attempt to fill this gap and provide a more detailed and accurate picture of the behavior of volatility of Central European stock index returns.

\section{Data Description}

To perform the analysis of stock returns on the Central European capital markets we focus on the 365-week period starting in January, 1996 and ending in December, 2002. We use weekly closing values of the value-weighted indices WIG, BUX and PX-50 of the Warsaw, Budapest and Prague stock exchanges, respectively. For an overview of the institutional framework, trading system, number of listed companies and other details on the Central European stock markets see Filer and Hanousek (1996) and Gilmore and McManus (2001). For comparison, we also perform the analysis on the DAX index of Deutsche Boerse. The data were downloaded from Bloomberg. 
The choice of weekly data is important for two reasons. First, the effect of nonsynchronous trading induces spurious autocorrelation into the index returns, but the lower the data frequency the less important this effect is (for details see Lo and MacKinlay, 1990). Thus to mitigate this effect we choose to use weekly rather than daily data. Second, almost all statistical inferences that will be drawn in the subsequent section are based on asymptotic theory and hence require a sufficient number of observations to be reliable. Using monthly data would therefore not be appropriate due to the short history of the stock markets under study.

We define the continuously compounded rate of return in week $t$ on an market index $\mathrm{Ml}$ as

$$
r_{t}^{\mathrm{MI}}=\operatorname{lnMI} \mathrm{MI}_{t}-\operatorname{lnMI} \mathrm{I}_{t-1}
$$

where $\mathrm{Ml}_{t}=\left\{\mathrm{WIG}_{t}, \mathrm{BUX}{ }_{t}, \mathrm{PX} 50_{t}, \mathrm{DAX}\right\}$. The descriptive statistics for the weekly returns on $\mathrm{W}, \mathrm{B}, \mathrm{P}$ and $\mathrm{D}$ are summarized in Table 1.

Table 1

Descriptive Statistics

\begin{tabular}{|l|c|c|c|c|}
\hline & WIG & BUX & PX/50 & DAX \\
\hline Mean & 0.00176 & 0.00449 & 0.00017 & 0.00063 \\
\hline Variance & 0.00167 & 0.00225 & 0.00093 & 0.00124 \\
\hline Maximum & 0.13470 & 0.14736 & 0.11580 & 0.12887 \\
\hline Minimum & -0.19244 & -0.33016 & -0.14045 & -0.14079 \\
\hline Skewness & -0.23585 & -1.06877 & -0.12109 & -0.36527 \\
\hline Kurtosis & 4.80766 & 11.67206 & 4.52814 & 4.45511 \\
\hline Jarque-Bera & $53.0793^{\text {** }}$ & $964.6577^{\text {** }}$ & $36.4067^{\text {** }}$ & $40.3178^{\text {** }}$ \\
\hline
\end{tabular}

Note: **significant at the $1 \%$ level.

The Jarque-Bera test indicates significant departures from normality for all indices. To learn more about the shape of the unconditional density function we employ a non-parametric kernel density estimator rather than reporting the usual histogram. Using Epanechnikov kernel and setting the bandwidth to the rule-of thumb value proposed by Silverman (1986) we obtain estimates of the unconditional densities given in Figures 1 and 2.

The estimated density functions of weekly returns on the Central European stock market indexes are similar to those usually obtained for indices of developed stock market (e.g. Fama, 1965). The empirical densities are leptokurtic which has important implications for further statistical tests and estimation. As we noted in earlier, the fat tails of the unconditional empirical distributions can be attributed to conditional heteroskedasticity and/or conditional non-normality. 
Figure 1

Kernel Density Estimates

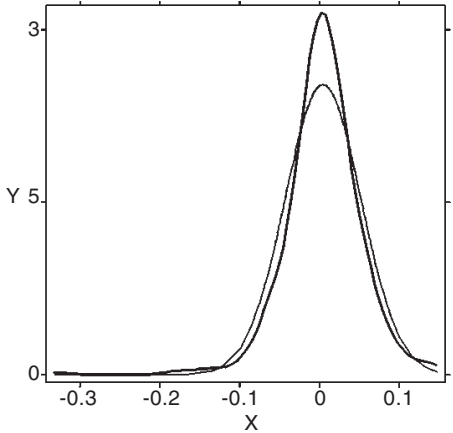

BUX

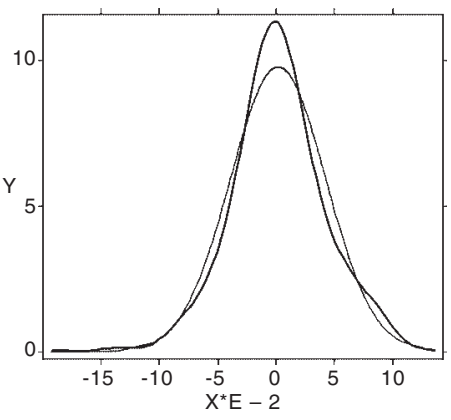

WIG

Figure 2

Kernel Density Estimates

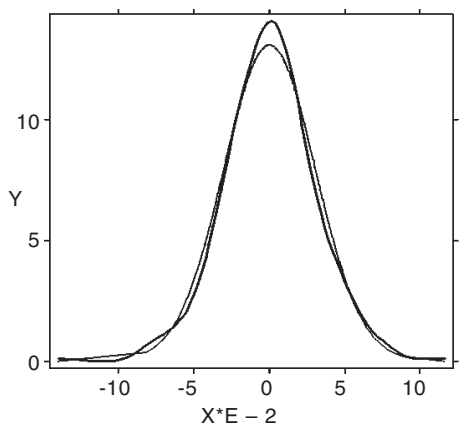

PX-50

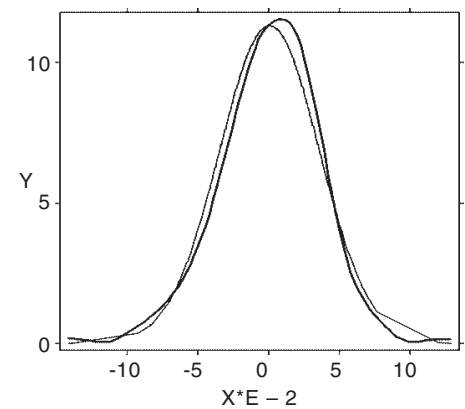

DAX

\section{Empirical Results}

This section reports the results of the analysis of stock returns performed on the Central European stock market indices. We start with the heteroskedasticity-consistent random walk test developed in Lo and MacKinlay (1988). Next come the results from ARIMA estimation (when applicable) followed by the ARCH-LM test results for conditional heteroskedasticity. Then we present the estimated GARCH-type models and the likelihood ratio tests for the null hypotheses that a) the residuals from the ARIMA models are conditionally Gaussian and b) the residuals are homoskedastic and unconditionally Student's $t$-distributed. We also examine the risk aversion of investors using the likelihood ratio test for the presence of the conditional volatility term in the mean equation for returns ( $\mathrm{ARCH}$-in-mean) and test for the asymmetric impact of shock to volatility (TARCH). Finally, we asses the ability of the proposed GARCH models to remove all non-linearity in the standardized residuals using the Ljung-Box $Q$ test and the BDS test.

Table 2 summarizes the results of the variance ratio test applied to the time series of weekly closing log-values of WIG, BUX, PX-50 and DAX indices. Following 
Lo and MacKinlay (1988), we compute the variance ratio's $\left(1+\widehat{\mathrm{M}}_{r}(q)\right)$ and the corresponding robust test statistics for $q=2,4,8,16$. The null hypothesis of random walk is decisively rejected for the Czech index PX-50. The variance ratio's for PX50 is larger than 1 for $q=2$ implying positive first-order autocorrelation in weekly returns on this index. The first-order autocorrelation coefficient is approximately equal to $11 \%$. In case of the Hungarian BUX index, the variance ratio for $q=8$ is statistically different from one at the $5 \%$ significance level, thereby rejecting the random walk null hypothesis. For WIG and DAX indices, the null hypothesis of random walk cannot be rejected.

Table 2

Random Walk Test Results

\begin{tabular}{|ll|r|r|r|l|}
\hline & & $q=2$ & $q=4$ & $q=8$ & $q=16$ \\
\hline \multirow{3}{*}{ WIG } & $1+\hat{\mathrm{M}}_{r}(q)$ & 0.997 & 1.032 & 1.106 & 1.003 \\
& $Z^{*}(q)$ & -0.057 & 0.384 & 0.931 & 0.023 \\
& Prob. & 0.954 & 0.701 & 0.352 & 0.982 \\
\hline \multirow{3}{*}{ BUX } & $1+\hat{\mathrm{M}}_{r}(q)$ & 0.964 & 1.162 & 1.229 & 1.202 \\
& $Z^{*}(q)$ & -0.532 & 1.445 & 2.209 & 1.117 \\
& Prob. & 0.595 & 0.149 & 0.027 & 0.264 \\
\hline \multirow{3}{*}{ PX-50 } & $1+\hat{\mathrm{M}}_{r}(q)$ & 1.108 & 1.306 & 1.430 & 1.266 \\
& $Z^{*}(q)$ & 1.902 & 3.722 & 3.901 & 1.622 \\
& Prob. & 0.057 & 0.000 & 0.000 & 0.105 \\
\multirow{3}{*}{ DAX } & $1+\hat{\mathrm{M}}_{r}(q)$ & 1.097 & 1.131 & 1.158 & 1.295 \\
& $Z^{*}(q)$ & 1.392 & 1.207 & 1.134 & 1.578 \\
& Prob. & 0.164 & 0.227 & 0.257 & 0.114 \\
\hline
\end{tabular}

The results of the random walk test indicate that the Czech and Hungarian stock contain predictable components. These findings are in contradiction to an earlier study due to Filer and Hanousek (1996) who were not able to reject the null hypothesis of random walk for PX-50, BUX and WIG using weekly data for the period starting at the data each index was first calculated and ending in June, 1996. Since our sample and that used by Filer and Hanousek are almost non-overlapping (they have only 24 common observations) it appears that predictability in the Czech and Hungarian stock returns has increased over the last decade. It may be interesting to divide our sample into two non-overlapping subsamples and run the random walk test independently in the subsamples. Our sample, however, contains only 365 weekly observations and thus the size of the subsamples would be small resulting into the variance ratio test having low power. For more on stock return predictability on Central European stock markets see Žikeš, 2003.

Since the random-walk model was rejected for the Czech and Hungarian stocks we will now search for a parametric model that would give us more insight into the nature and degree of predictability. We do this by identifying and estimating an $\operatorname{ARIMA}(p, 1, q)$ model using the Box-Jenkins methodology. For the PX-50 index we find an ARIMA $(1,1,1)$ model to best describe the data generating process and for BUX index, ARIMA $(2,1,0)$ appears to be the best alternative. We do not report the estimation output here since both models will be re-estimated allowing for ARCH effects in the residuals in the subsequent paragraph. ${ }^{1)}$ Next we run the ARCH-LM

1) The estimation outputs are available from the authors upon request. 
test for the null hypothesis of no conditional heteroskedasticity in the residuals from the ARIMA models. For WIG and DAX indices, we apply the test to the residuals from a regression of returns on a constant only, since for these indices the null hypothesis of random walk could not be rejected (e.g. the residuals should approximate a white noise sequence). We set the order of the test, $p=8$. Also we run the LjungBox $Q$-test of order eight on the estimated squared residuals. Table 3 summarizes the results.

Table 3

\section{ARCH-LM Test Results}

\begin{tabular}{|l|c|c|c|c|}
\hline & WIG & BUX & PX-50 & DAX \\
\hline ARCH-LM Test Stat. & 39.669 & 12.560 & 43.843 & 73.030 \\
\hline Prob. & 0.0000 & 0.1279 & 0.0000 & 0.0000 \\
\hline Ljung-Box Q-stat. & 45.557 & 13.791 & 45.511 & 107.76 \\
\hline Prob. & 0.0000 & 0.0000 & 0.0000 & 0.0000 \\
\hline
\end{tabular}

Except for the Hungarian BUX index, both tests clearly indicate presence of conditional heteroskedasticity in the estimated residuals. In case of the BUX index the null hypothesis of homoskedasticity is rejected at the $10 \%$ significance level using the Ljung-Box $Q$-test but the ARCH-LM test fails to reject the null at the conventional significance levels. Hence the presence of conditional heteroskedasticity in the residuals from the ARIMA $(2,1,0)$ model for BUX index has to be further investigated.

We now turn to modelling conditional heteroskedasticity in the index returns. In particular, we assume the GARCH $(1,1)$ model for the residuals from the corresponding mean regression equations. It turns out that this model has superior performance in comparison to other GARCH specifications. Thus for the Czech PX-50 index we estimate an ARIMA $(1,1,1)-\mathrm{GARCH}(1,1)$ model, for the BUX index an ARIMA $(2,1,0)$-GARCH $(1,1)$ model and for WIG and DAX indices we assume a random walk model whose increments obey a $\mathrm{GARCH}(1,1)$ process. We estimate these models by the method of maximum likelihood under the assumption that the residuals are Student- $t$ distributed with $v$ degrees of freedom. ${ }^{2}$ The estimated coefficients are asymptotically normally distributed. Table 4 a reports the estimated random walk model with conditionally heteroskedastic increments for the Polish WIG index.

Table 4a

GARCH (1,1)- $t$ Model for WIG

\begin{tabular}{|c|c|c|c|c|}
\hline & Coefficient & Std. error & $z$-stat. & Probability \\
\hline$\mu$ & $7.66 .10^{-4}$ & $1.90 .10^{-3}$ & 0.403 & 0.687 \\
\hline$\Phi$ & $1.04 .10^{-4}$ & $0.92 .10^{-5}$ & 1.125 & 0.260 \\
\hline$\delta_{1}$ & 0.854 & 0.089 & 9.631 & 0.000 \\
\hline$\alpha_{1}$ & 0.082 & 0.045 & 1.823 & 0.068 \\
\hline$v$ & 6.521 & 2.627 & n.m. & n.m. \\
\hline
\end{tabular}

2) The $\mathrm{BHHH}$ algorithm is employed to maximize the likelihood function. 
The estimated GARCH coefficients $\delta_{1}$ and $\alpha_{1}$ are significant at the $1 \%$ and $10 \%$ level, respectively and their sum is less then one implying that the GARCH model is stationary, thought the volatility is fairly persistent since $\left(\delta_{1}+\alpha_{1}\right)$ is close to one. The estimated number of degrees of freedom of the conditional $t$-distribution is equal to 6.5 which suggests that the returns on the WIG index are conditionally non-normally distributed. To summarize, both heteroskedasticity and non-normality account for the fat tails in the empirical distribution of the returns on WIG.

Table 4b

GARCH (1,1)- $t$ Model for BUX

\begin{tabular}{|c|c|c|c|c|}
\hline & Coefficient & Std. error & $z$-stat. & Probability \\
\hline$\mu$ & 0.005 & 0.002 & 2.453 & 0.014 \\
\hline$\phi_{2}$ & 0.133 & 0.049 & 2.684 & 0.007 \\
\hline$\Phi$ & $1.34 .10^{-4}$ & $8.18 .10^{-5}$ & 1.643 & 0.100 \\
\hline$\delta_{1}$ & 0.765 & 0.108 & 7.101 & 0.000 \\
\hline$\alpha_{1}$ & 0.060 & 0.032 & 1.863 & 0.062 \\
\hline$v$ & 4.218 & 0.964 & n.m. & n.m. \\
\hline
\end{tabular}

$$
R^{2}=0.034
$$

Recall that both the Ljung-Box $Q$ test for serial correlation in the squared residuals and the ARCH-LM test failed to reject the null hypothesis of no ARCH effects in the residuals from the ARIMA $(2,1,0)$ model for the Hungarian BUX index. From the estimation output, reported in Table $4 \mathrm{~b}$ we see, however, that the GARCH coefficients $\delta_{1}$ and $\alpha_{1}$ are statistically significant at the $1 \%$ and $10 \%$ level, respectively. The estimated number of degrees of freedom is equal to 4.2 which is a very small value given the fact that for $v \leq 4$ the kurtosis of a Student- $t$ random variable with $v$ degrees of freedom is infinite. But the low value of $\hat{v}$ is not surprising since the empirical kurtosis of the BUX returns is quite high, equal to 11.67. Thus, as with the WIG index, the leptokurtosis in the empirical distribution function of the returns on the BUX index is induced by both heteroskedasticity and non-normality.

Table 4c

GARCH (1,1)-t Model for PX-50

\begin{tabular}{|c|c|c|c|c|}
\hline & Coefficient & Std. error & $z$-stat. & Probability \\
\hline$\mu$ & $1.27 .10^{-4}$ & $7.23 .10^{-4}$ & 0.176 & 0.860 \\
\hline$\phi_{1}$ & 0.633 & 0.224 & 2.823 & 0.005 \\
\hline$\theta_{1}$ & 0.508 & 0.254 & 1.996 & 0.046 \\
\hline$\varpi$ & $1.95 .10^{-5}$ & $2.19 .10^{-5}$ & 0.887 & 0.375 \\
\hline$\delta_{1}$ & 0.928 & 0.045 & 20.36 & 0.000 \\
\hline$\alpha_{1}$ & 0.055 & 0.030 & 1.812 & 0.069 \\
\hline$v$ & 7.238 & 3.475 & n.m. & n.m. \\
\hline
\end{tabular}

$R^{2}=0.021$ 
In Table 4c, there is summarized the estimated ARIMA $(1,1,1)-\mathrm{GARCH}(1,1)$ model for the Czech index, PX-50. We obtain very similar results to those for WIG and BUX in that the GARCH parameters $\delta_{1}$ and $\alpha_{1}$ are statistically significant at the $1 \%$ and $10 \%$ level, respectively, and $\hat{v}$ is much less than ten. Note that although the $\mathrm{GARCH}(1,1)$ model is stationary, $\delta_{1}+\alpha_{1}=0.983$, i.e. the volatility of the returns on $\mathrm{PX}-50$ is very persistent.

Table 4d

GARCH (1,1)- $t$ Model for DAX

\begin{tabular}{|c|c|c|c|c|}
\hline & Coefficient & Std. error & $z$-stat. & Probability \\
\hline$\mu$ & 0.004 & 0.002 & 2.319 & 0.020 \\
\hline$\Phi$ & $3.14 .10^{-5}$ & $2.58 .10^{-5}$ & 1.219 & 0.223 \\
\hline$\delta_{1}$ & 0.807 & 0.053 & 15.30 & 0.000 \\
\hline$\alpha_{1}$ & 0.186 & 0.053 & 3.531 & 0.001 \\
\hline$v$ & 18.62 & 12.74 & n.m. & n.m. \\
\hline
\end{tabular}

Finally, we examine the German index DAX (see Table 4d.). Surprisingly, the estimated number of degrees of freedom of the conditional Student- $t$ distribution for returns is quite large compared to those for the market indices of the Central European stock markets. It falls into the inconclusive region defined in Connolly (1992) and hence it is difficult to comment on the distributional properties of returns without further testing. Intuitively, since the GARCH parameters are highly significant and the volatility of returns is very persistent $\left(\delta_{1}+\alpha_{1}=0.993\right)$ it may be the case that the excess kurtosis in the DAX returns is induced primarily by conditional heteroskedasticity. To test this hypothesis rigorously, we employ a likelihood ratio test.

The Table 5 presents the likelihood ratios for a) the null hypothesis that the returns on the corresponding market indices are conditionally normally distributed $(1 / v=0)$ and $b)$ the null hypothesis of unconditionally Student- $t$ distributed returns with $v$ degrees of freedom $\left(\alpha_{1}=\delta_{1}=0\right)$. The likelihood ratios are asymptotically $\chi^{2}$-distributed with a) one, and b) two degrees of freedom. The $5 \%$ critical values are thus 3.84 and 5.99, respectively. Note, however, that according to Bollerslev (1987) the distribution of the test statistic $\mathrm{LR}_{1 / v=0}$ in samples of moderate sizes tends to be more concentrated towards the origin than the $\chi_{1}^{2}$-distribution and hence a test based on the 3.84 critical value has lower size (is more conservative). Bollerslev(1987) provides the appropriate $5 \%$ critical value, 2.71 .

Table 5

\section{LR Test Results}

\begin{tabular}{|l|c|c|c|}
\hline & $\hat{v}$ & $\mathrm{LR}_{1 / v=0}$ & LR $_{\alpha_{1}=\delta_{1}=0}$ \\
\hline WIG & 6.521 & $11.50^{*}$ & $16.04^{*}$ \\
\hline BUX & 4.218 & $52.97^{*}$ & $16.52^{*}$ \\
\hline PX-50 & 7.238 & $7.82^{*}$ & $13.76^{*}$ \\
\hline DAX & 18.62 & 2.35 & $61.37^{*}$ \\
\hline
\end{tabular}

Note: *significant at the $1 \%$ level. 
The null hypothesis of unconditionally Student- $t$ distributed is decisively rejected for all four market indices. These findings are in line with our estimates of the $\mathrm{GARCH}$ parameters: they all were statistically significant. Hence, conditional normality is rejected for the Central European stock markets indices. The likelihood ratio test fails to reject the null for the German DAX. Again, these results are in accordance with our estimates of $v$ : the smaller the $\hat{v}$, the larger the likelihood ratio for the null hypothesis of conditional normality. To summarize, on the basis of the GARCH- $t$ model and the likelihood ratios we have found that in case of the returns on the Central European stock market indices, both conditional heteroskedasticity and non-normality accounts for excess kurtosis in the empirical distribution functions of index returns. For the German index DAX, the picture is less clear cut, though it appears that the primary source of the 'fat tails' in the empirical distribution is timevarying volatility.

Next we test the simple GARCH model against two alternative specifications. First, we considered an asymmetric impact of positive and negative shocks to returns on their expected volatility (TARCH). Second, we included the expected volatility term, $h_{t}$, into the mean equation for returns (ARCH-in-mean). Both alternative specifications can be tested using the usual likelihood ratio. Table 6 reports the likelihood ratios for a) the null hypothesis that the shocks to returns have a symmetric effect on future volatility $(\lambda=0)$ and $b$ ) the null hypothesis of risk-neutrality of investors ( $\pi=$ $0)$. Under both null hypotheses the likelihood ratios are asymptotically $\chi^{2}$ distributed with one degree of freedom, hence the $5 \%$ critical value is equal to 3.84 .

Table 6

LR Test Results

\begin{tabular}{|l|c|c|}
\hline & $\mathrm{LR}_{\lambda=0}$ & $\mathrm{LR}_{\pi=0}$ \\
\hline WIG & 1.30 & $6.01^{*}$ \\
\hline BUX & n.m. & 2.21 \\
\hline PX-50 & 3.10 & 0.07 \\
\hline DAX & n.m. & 0.01 \\
\hline
\end{tabular}

Note: *significant at the $5 \%$ level.

Note that we do not report the values of $L R_{\lambda=0}$ for BUX and DAX indices because the estimates of the constant term $\varpi$ in the TARCH variance equation are negative which violates the assumption that all GARCH coefficients are positive (a negative

Table 7

GARCH-M- $t$ Model for WIG

\begin{tabular}{|c|c|c|c|c|}
\hline & Coefficient & Std. error & $z$-stat. & Probability \\
\hline$\mu$ & -0.030 & 0.0144 & -2.077 & 0.038 \\
\hline$\pi$ & 0.817 & 0.377 & 2.167 & 0.030 \\
\hline$\varpi$ & $8.53 .10^{-5}$ & $6.42 .10^{-5}$ & 1.328 & 0.184 \\
\hline$\delta_{1}$ & 0.875 & 0.060 & 14.49 & 0.000 \\
\hline$\alpha_{1}$ & 0.071 & 0.033 & 2.136 & 0.033 \\
\hline$v$ & 6.406 & 2.457 & n.m. & n.m. \\
\hline
\end{tabular}


$\varpi$ may cause $h_{t}$ to be negative for some $t$ which is not consistent with the non-negativity property of variance). For WIG and PX-50 the null hypothesis that the shocks to returns have symmetric impact on volatility cannot be rejected on the conventional significance levels. Turning to the null hypothesis of risk-neutrality, the likelihood ratio test fails to reject the null for BUX, PX-50 and DAX, but does reject it at the $1 \%$ significance level for the Polish WIG index.

Since the null hypothesis of risk neutrality was rejected in case of the WIG index, we now estimate the $\mathrm{ARCH}$-in-mean model assuming Student- $t$ distribution of the error terms. Table 7 summarizes the estimation output. The estimate of $\pi$, which measures the degree of risk aversion, is positive and significant at the $5 \%$ level. It implies that the investors trading on the Polish stock market are risk-averse: they require higher returns in periods when they expect higher volatility (risk). ${ }^{3)}$ The fact that expected volatility, $h_{t}$, is significant in the mean equation has also important implications for return predictability. To see this, recall that $h_{t}$ is a function of past squared residuals from the mean equation. The expected return on the WIG index for period $t+1$ based on the information available in period $t$, can be thus written as

$$
\begin{aligned}
E\left[r_{t+1} \mid \Phi_{t}\right] & =\mu+\pi h_{t+1}, \\
& =\mu+\pi\left(\varpi+\delta_{1} h_{t}+\alpha_{1} \varepsilon_{t}^{2}\right), \\
& =\mu+\pi\left(\zeta+\psi(L) \varepsilon_{t}^{2}\right),
\end{aligned}
$$

where $\psi(L)$ is an infinite-order polynomial in the lag operator. ${ }^{4)}$ Now because both $h_{t}$ and $\varepsilon_{t}^{2}$ are known at time $t$ the return for period $t+1$ is predictable from the time

Table 8

Diagnostic Tests

\begin{tabular}{|l|c|c|c|c|}
\hline & WIG & BUX & PX-50 & DAX \\
\hline$Q(4)$ & 2.27 & 2.46 & 3.92 & 6.39 \\
\hline$Q(8)$ & 3.95 & 5.11 & 6.99 & 11.91 \\
\hline$Q(12)$ & 12.53 & 7.72 & 9.47 & 19.05 \\
\hline$Q^{2}(4)$ & 2.39 & 2.54 & 2.07 & 1.60 \\
\hline$Q^{2}(8)$ & 11.73 & 4.26 & $16.57^{*}$ & 4.67 \\
\hline$Q^{2}(12)$ & 15.23 & 5.62 & $20.64^{*}$ & 4.93 \\
\hline $\operatorname{BDS}(2)$ & -0.97 & -0.51 & 0.35 & 0.58 \\
\hline $\operatorname{BDS}(4)$ & 0.45 & -0.08 & -1.14 & 0.09 \\
\hline $\operatorname{BDS}(6)$ & 1.08 & 0.83 & $-1.97^{*}$ & 0.57 \\
\hline
\end{tabular}

Note: *significant at the $5 \%$ level.

3) There has been evidence in the literature on the ARCH-in-mean models of the sensitivity of the parameter estimates to the distributional assumptions made to obtain the estimates by MLE (see Bollerslev et.at., 1992, for an overview). It has been reported, for example, that by changing the conditional distribution from normal to Student- $t$, the estimated $\pi$ dropped substantially and was no-longer significant. When we use normal distribution to estimate the ARCH-in-mean model for the WIG index, we obtain $\hat{\pi}=7.73$ with (Bollerslev and Woodridge, 1991 ) robust $p$-value equal to 0.087 . Thus the sensitivity observed on developed markets carries over to the Polish stock market. $\mathrm{ARCH}$.

4) We assume that $\delta_{1}<1$ and hence the GARCH $(1,1)$ process can be expressed as an infinite order 
series of historical volatility and past squared residuals. The conclusion that WIG's returns are predictable is in contradiction to the result of the robust variance ratio test for the null hypothesis of random walk. But this should not be surprising. The variance ratio test, by construction, has power only against linear dependencies in the time series of returns. In an $\mathrm{ARCH}$-in-mean model there is a correlation between current return and lagged squared disturbances. It follows that the variance ratio test cannot detect such non-linearities and hence fails to reject the null hypothesis of random walk. ${ }^{5}$ ) The $\mathrm{ARCH}$-in-mean model therefore provides us with an important insight into the time-series predictability of stock returns.

Finally, having estimated the corresponding ARIMA-GARCH models for the studied stock market indices, we should perform diagnostic testing to asses whether the models are correctly specified. We start by testing the standardized residuals for serial correlation. This may seem superfluous because the ARIMA models were specified in a way that would remove possibly all autocorrelation in the residuals. But because we did not report the estimates and diagnostic checks before for the sake of brevity, we should do it now. The first three rows of Table 8 therefore report the Ljung-Box $Q$ statistics applied to the standardize residuals from the models of Tables $4 b, c, d$ and 7 . Clearly, the null hypothesis of no serial correlation cannot be rejected on the conventional significance levels and hence the mean equations appear to be correctly specified. Next we run the Ljung-Box $Q$ test on the squared standardized residuals (we label the test statistic $Q^{2}$ to emphasize that the test is run on squared standardized residuals). The null hypothesis of no serial correlation in squared standardized residuals can be rejected only for the PX-50 index. Hence the $\mathrm{GARCH}(1,1)$ model in this case fails to appropriately fit the volatility process. No reasonable alternative specifications, however, were found to perform better. ${ }^{6)}$ Finally, we apply the BDS test on the standardized residuals to determine, whether the $\mathrm{GARCH}$ model removes all non-linearities in the time-series of returns. We set the proximity parameter to 0.5 and run the test for embedding dimensions 2, 4, and 6 . The null hypothesis that the standardized residuals are independently identically distributed can be rejected at $5 \%$ for PX-50 index only, which is consistent with the findings of the Ljung-Box $Q$ test. To summarize, the diagnostic checks performed on the standardized residuals provide convincing evidence that, except for the PX-50 index, the ARIMA-GARCH models were appropriately specified and can thus be used for rational predictions of both index returns and their volatility.

\section{Conclusion}

The aim of this paper has been to investigate the behavior of volatility and the distributional properties of the Central European stock index returns. Using weekly data for the period 1996:1 through 2002:12, we focused on the Czech, Hungarian and Polish stock markets and considered the German market as a benchmark. We extended the previous empirical work by explicitly modelling the 'fat tails' in the un-

5) To detect non-linearities in stock returns, we could also apply the BDS test to the returns. But since we have seen that the stock returns are heteroskedastic the result of the BDS test would be necessarily ambiguous: we would not know whether we should attribute the rejection of the iid null hypothesis to nonlinearities in the mean or non-linearities in the variance.

6) The correlogram of the squared standardized residuals reveals that there is a significant spike in both the autocorrelation and partial autocorrelation functions at lag 7 . This indicates that a $\mathrm{GARCH}(1,8)$ may better fit the data. Some of the estimated coefficients in this model are, however, negative, which is ruled out by assumption. The estimates are available from the author upon request. 
conditional distributions of stock returns using a GARCH-type model with Student- $t$ distributed disturbances. To briefly summarize our analysis, we found that:

- the Central European stock index prices do not follow random walk,

- stock index returns are predictable from the time-series of historical returns using a linear (ARMA) or non-linear (ARCH-in-mean) parametric model,

- stock return volatility changes over time and can be well described by a GARCH-type model,

- empirical density functions of stock returns exhibit excess kurtosis that can be attributed to both conditional heteroskedasticity and non-normality.

We hasten to emphasize that the predictability of stock returns need not be a symptom of market inefficiency. Even in an efficient market stock returns may be correlated simply because the expected return systematically changes over time. But our analysis did not study the way expected return is determined at all and hence any conclusion regarding the EMH is impossible.

Our empirical findings have also important implications for asset pricing. Consider, for simplicity, the well-known Black-Scholes model for pricing European style call options on a non-dividend paying stock. This model is derived under three crucial assumptions: a) log-stock prices follow random walk, b) returns are normally distributed, and c) volatility is constant through time. But none of these assumptions is satisfied empirically, so the Black-Scholes cannot be used for rational pricing of options on the Central European stock market indices.

\section{References}

Bollerslev, T. P. (1986), "Generalized Autoregressive Conditional Heteroskedasticity." Journal of Econometrics, 31, pp. 309-328.

(1987), "A Conditionally Heteroskedastic Time Series Model for Speculative Prices and Rates of Return." The Review of Economics and Statistics, 69, pp. 542-547.

Bollerslev, T., Chou, R., Kroner, K. (1992), "ARCH Modelling in Finance." Journal of Econometrics, 52, pp. 5-59.

Bollerslev, T. P., Wooldridge, J. M. (1992), "Quasi-Maximum Likelihood Estimation and Inference in Dynamic Models with Time Varying Covariances." Econometric Reviews, 11, pp. 143-172.

Brock, W., Dechert, W., Scheinkman, J. (1987), "A Test for Independence Based on the Correlation Dimension." University of Wisconsin at Madison, Department of Economics, Working Paper.

Cochrane, J. H. (2001), Asset Pricing. Princeton: Princeton University Press.

Connolly, R. A. (1989), "An Examination of the Robustness of the Weekend Effect." Journal of Financial and Quantitative Analysis, 24, pp. 133-169.

Engle, R., Lilien, D. M., Robins, R. P. (1987), "Estimating Time-Varying Risk Premia in the Term Structure: The ARCH-M Model.“ Econometrica, 55, pp. 391-407.

Fama, E. F. (1965), "The Behavior of Stock Market Prices.“ Journal of Business, 38, pp. 34-105.

Filer, R. K., Hanousek, J. (1996), "The Extent of Efficiency in Central European Equity Markets." Center for Economic Research and Graduate Education, Charles University, Prague, Working Paper.

Gilmore, C. G., McManus, G. M. (2001), "Random-Walk and Efficiency Tests of Central European Equity Markets." European Financial Management Association Conference, Lugano.

Glosten, L. R., Jaganathan, R., Runkle, D. (1993), "On the Relation between the Expected Value and Volatility of the Normal Excess Return on Stocks." Journal of Finance, 48, pp. 1779-1801.

Lo, A. W., MacKinlay, A. C. (1988), "Stock Prices Do Not Follow Random Walk: Evidence from a Simple Specification Test." Review of Financial Studies, 1, pp. 41-66.

of Econometrics, 45, pp. 181-211.

(1990), "An Econometric Analysis of Nonsynchronous Trading." Journal

Mandelbrot, B. (1963), "The Variation of Certain Speculative Prices.“ Journal of Business, 36, pp. 394-419. 
Silverman, B. W. (1986), Density Estimation for Statistics and Data Analysis. New York: Chapman and Hall.

Vošvrda, M., Filáček, J., Kapička, M. (1998), "The Efficient Market Hypothesis and Volatility: the Case of Prague Stock Exchange." Bulletin of the Czech Econometric Society, 7, pp. 55-67.

Zakoian, J. M. (1994), "Threshold Heteroskedastic Models." Journal of Economic Dynamics and Control, 18, pp. 931-944.

Žikeš, F. (2003), "The Predictability of Asset Returns: An Empirical Analysis of Central European Stock Markets." Charles University, Prague, Master Thesis (http://ies.fsv.cuni.cz/knih/papers/zikes.pdf). 